\title{
ESTABILIDADE DE AGREGADOS DE UM LATOSSOLO VERMELHO DEGRADADO EM RECUPERAÇÃO COM ADUBOS VERDES, CALCÁRIO E GESSO ${ }^{(1)}$
}

\author{
Carolina dos Santos Batista Bonini ${ }^{(2)} \&$ Marlene Cristina Alves ${ }^{(3)}$
}

\begin{abstract}
RESUMO
A utilização incorreta dos solos e as grandes construções no meio rural vêm causando alterações no ambiente edafoclimático, o que torna os solos menos produtivos, aumentando assim as áreas degradadas. Tendo em vista esse problema, o presente trabalho teve como objetivo avaliar a recuperação de um Latossolo Vermelho que se encontra há 17 anos com o uso de adubos verdes, calcário, gesso e braquiária. Foi utilizada a qualidade da estrutura como indicadora da recuperação do solo. O trabalho foi desenvolvido em delineamento experimental inteiramente casualizado, com nove tratamentos e quatro repetições. Os tratamentos foram: testemunha (solo mobilizado) até 1999, sendo depois implantada Brachiaria decumbens; mucuna-preta (Stizolobium aterrimum Piper \& Tracy) até 1999, substituída por B. decumbens; guandu (Cajanus cajan (L.) Millsp) até 1994, substituído por feijão-de-porco (Canavalia ensiformis (L.)) e, a partir de 1999, por B. decumbens; calcário+mucuna-preta até 1999 , substituído por $B$. decumbens; calcário+guandu até 1994, substituído por feijão-de-porco e, a partir de 1999, por $B$. decumbens; calcário+gesso+mucuna-preta até 1999, substituído por B. decumbens; calcário+gesso+guandu até 1994, substituído por feijão-de-porco e, partir de 1999, por B. decumbens; e duas testemunhas: solo exposto (sem técnica de recuperação) e vegetação nativa de Cerrado. Foi avaliada a estabilidade de agregados em água e o conteúdo de matéria orgânica nas camadas de 0-10, 10-20 e 20-40 cm. O solo vem sendo recuperado, e a qualidade da estrutura, comparada à condição natural de Cerrado, na camada de $0-10 \mathrm{~cm}$ foi mais alterada positivamente; os tratamentos
\end{abstract}

\footnotetext{
${ }^{(1)}$ Parte do trabalho de Dissertação de Mestrado desenvolvido no Programa de Pós-Graduação em Sistemas de Produção da Faculdade de Engenharia de Ilha Solteira - UNESP, Campus de Ilha Solteira. Recebido para publicação em 22 de dezembro de 2009 e aprovado em 16 de maio de 2011.

(2) Mestranda da Faculdade de Engenharia, Universidade Estadual Paulista, Campus de Ilha Solteira. Bolsista da Fapesp. E-mail: carolsbatistabonini@hotmail.com

(3) Professora Titular, Universidade Estadual Paulista, Campus de Ilha Solteira, Departamento de Fitossanidade, Engenharia Rural e Solos. CEP 15385-000 Ilha Solteira (SP). Bolsista de Produtividade em Pesquisa/CNPq. E-mail: mcalves@agr.feis.unesp.br
} 
que receberam calcário e gesso foram os melhores, e o teor de matéria orgânica nessa camada foi superior ao das demais. Abaixo da camada superficial do solo, os tratamentos estão agindo de forma semelhante. $O$ solo exposto, em todas as camadas, apresentou-se com má qualidade da estrutura, avaliada pela estabilidade de agregados e pelo diâmetro médio ponderado.

Termos de indexação: degradação do solo, estrutura do solo, matéria orgânica.

\title{
SUMMARY: AGGREGATE STABILITY OF A DEGRADED OXISOL IN RECOVERY WITH GREEN MANURE, LIME AND GYPSUM
}

\begin{abstract}
Inadequate land use and large buildings in rural areas are causing damages to the soilclimate environment, reducing productivity and thus expanding degraded areas. In view of this problem, this study evaluated the recovery of an Oxisol that had been treated with green manure, soil amendment, gypsum and pasture in the last 17 years. The quality of the structure was used as an indicator of soil recovery. The study was conducted in a completely randomized design with nine treatments and four replications. The treatments consisted of a control (mobilized soil) until 1999 and then planted with signal grass (Brachiaria decumbens); black velvet bean (Stizolobium aterrimum) (Piper \& Tracy) until 1999, then replaced by B. decumbens; pigeon pea (Cajanus cajan (L.)) until 1994, then replaced by jack bean (Canavalia ensiformis (L.)); pigeon pea (Cajanus cajan $(L)$.$) until 1994, then replaced by signal grass (B.$ decumbens); Lime + S. aterrimum until 1999 when replaced by B. decumbens; Lime + C. cajan until 1994, when replaced by $\mathrm{C}$. ensiformis and after 1999 replaced by $\mathrm{B}$. decumbens; Lime + Gypsum +S. aterrimum until 1999, when replaced by B. decumbens; Lime + Gypsum $+\mathrm{C}$. cajan until 1994, when replaced by C. ensiformis and after 1999 replaced by $\mathrm{B}$. decumbens, and two controls: bare soil (without recovery techniques) and native Cerrado vegetation (savannah). Water aggregate stability and soil organic matter were evaluated in the layers 0 10; 10-20 and 20-40 cm. The soil is being recovered and the structure quality, compared to the native Cerrado, was most improved in the 0-10 cm layer; the results of the treatments with lime and gypsum were the best and soil organic matter in this layer was higher than in the others. Below the surface layer, the effect of the treatments was similar. The structure quality of the bare soil, measured by aggregate stability and mean weighted diameter, was poor in all layers.
\end{abstract}

Index terms: degraded soil, soil structure, organic matter.

\section{INTRODUÇÃO}

No planejamento de recuperação de uma área degradada, o grande desafio a ser alcançado é o estabelecimento de um horizonte A, para que a partir daí o processo seja catalisado pela biosfera, podendo surgir outros horizontes, conforme o condicionamento natural. Assim, estará se interferindo em um ou mais fatores de formação do solo, numa tentativa de acelerar sua gênese (Kitamura et al., 2008). Portanto, em trabalho de recuperação, a primeira atividade compreende a identificação e caracterização dos processos de degradação atuantes e a análise de suas consequências ambientais. Para isso, é necessário o uso de indicadores que traduzam quantitativa ou qualitativamente o grau da degradação e, ainda, permitam estimar a dimensão dos esforços técnicos e econômicos que deverão ser alocados na recuperação (Bitar, 1997).
Os relatos na literatura sobre indicadores da qualidade do solo são divergentes. Numa recente revisão sobre a contribuição da boa qualidade do solo para a saúde ambiental, Jaenicke (2001) conclui que a qualidade do solo continua a receber pouca atenção, embora haja maior conscientização dos potenciais beneficios da definição de indicadores de qualidade. Salienta também que existem brechas significativas em nossa habilidade para identificar as relações físicas, químicas e ecológicas necessárias para avaliar a qualidade ambiental.

Roth et al. (1991) constataram que os sistemas de manejo que mantêm o solo descoberto diminuem a estabilidade de agregados, causando selamento superficial, reduzindo a infiltração de água e, consequentemente, induzindo à enxurrada e à erosão. Por isso, a estabilidade de agregados tem sido usada como indicador da qualidade física do solo, pois é sensível às alterações, conforme o manejo adotado. 
A adição de várias fontes de matéria orgânica tem sido utilizada com o objetivo de melhorar as propriedades do solo. O fornecimento contínuo de material orgânico pela serrapilheira e, ou, por excreções radiculares, cujos subprodutos são constituídos por moléculas orgânicas em diversas fases de decomposição, atua como agente de formação e estabilização dos agregados, proporcionando melhor estruturação do solo (Campos et al., 1995; Campos \& Alves, 2008). Campos (2006) e Taboada-Castro et al. (2009) desenvolveram pesquisas para avaliar os efeitos de matéria orgânica na recuperação de áreas degradadas e verificaram o aumento do diâmetro médio ponderado de agregados na camada superficial.

Os efeitos promovidos pela adubação verde nas propriedades químicas do solo são diversificados, dependendo da espécie utilizada, do manejo dado à massa vegetal, da época de semeadura, do corte do adubo verde, do tempo de permanência dos resíduos no solo, das condições locais e das interações desses fatores (Alcântara et al., 2000). Avaretto et al. (2000) mencionam que as culturas forrageiras, gramíneas e leguminosas, em função de suas características (proteger o solo do impacto da água das chuvas e fornecer matéria orgânica), têm sido utilizadas em programas de recuperação de áreas degradadas.

A presença de agregados estáveis potencializa a capacidade de armazenamento de água, diminuindo as perdas de partículas e nutrientes por processos erosivos, e facilita a proteção física e o acúmulo de matéria orgânica no solo (Jastrow et al., 1998).

O objetivo deste trabalho foi avaliar a recuperação de um Latossolo Vermelho submetido há 17 anos a manejos agrícolas utilizando-se adubos verdes, calcário, gesso e braquiária. Foi utilizado o desenvolvimento da estrutura como indicador da recuperação do solo.

\section{MATERIAL E MÉTODOS}

O experimento foi conduzido no município de Selvíria, MS, Brasil, numa área localizada na margem direita do rio Paraná, apresentando as coordenadas geográficas de $51^{\circ} 22$ ' de longitude oeste de Greenwich e $20^{\circ} 22^{\prime}$ 'de latitude sul, com altitude de $327 \mathrm{~m}$. As médias anuais de precipitação pluvial, temperatura e umidade relativa do ar da área são de $1.370 \mathrm{~mm}$, $23,5^{\circ} \mathrm{C}$ e $70-80 \%$, respectivamente.

O solo da área de estudo foi classificado como Typic Acrustox (Soil Survey Staff, 1999), Ferralsol (FAO, 1998) ou Latossolo Vermelho distrófico (Demattê, 1980; Embrapa, 2006), textura franco-argilo arenosa (Kitamura, 2007), muito profundo, rico em oxihidróxidos de $\mathrm{Fe}$ e $\mathrm{Al}$. A sua fração argila é de baixa atividade e composta essencialmente por gibbsita e caulinita.

Foram retirados da área em estudo, em média, 8,6 m da camada superficial do perfil do solo, para uso na construção da Usina de Ilha Solteira. A obra teve início em 1969; portanto, o subsolo da área em estudo está exposto há 40 anos. Em 1992, o subsolo exposto apresentava-se com compactação superficial e baixa presença de vegetação espontânea. Nesse mesmo ano iniciou-se o trabalho de recuperação, e o preparo do solo foi realizado efetuando-se uma subsolagem, atingindo profundidade média de $40 \mathrm{~cm}$. Foi efetuada também uma aração e, após essa operação, uma gradagem niveladora. Foi realizada em 1992, na camada de 0-20 cm, a caracterização física e química do solo (Quadro 1).

A correção do solo, efetuada em 1992, foi baseada na caracterização química da área experimental. A aplicação de calcário, para elevar a saturação por bases a $70 \%$, foi feita empregando-se a quantidade de $18,5 \mathrm{~kg}$ de calcário por parcela (parcelas com calcário somente); no caso das parcelas com calcário e gesso, houve substituição de $25 \%$ de $\mathrm{CaO}$ do calcário pelo equivalente a $18,5 \%$ de $\mathrm{Ca}$ do gesso, aplicando-se $5,2 \mathrm{~kg}$ de gesso e 13,8 kg de calcário por parcela, ambos incorporados ao solo. Foi utilizado calcário dolomítico com PRNT de 70 \%. Cada parcela teve dimensão de $10 \times 10 \mathrm{~m}$.

Os adubos verdes foram semeados em linha, manualmente e em cada parcela, em dezembro-janeiro dos anos de 1992 a 1996. A densidade de semeadura

Quadro 1. Macroporosidade, microporosidade, porosidade total e densidade do solo e atributos químicos do subsolo exposto, caracterizado antes da implantação do experimento (1992)

\begin{tabular}{|c|c|c|c|c|c|c|c|c|c|c|}
\hline Profundidade & \multicolumn{2}{|c|}{ Macroporosidade } & \multicolumn{3}{|c|}{ Microporosidade } & \multicolumn{2}{|c|}{ Porosidade total } & \multicolumn{3}{|c|}{ Densidade do solo } \\
\hline $\mathrm{cm}$ & \multicolumn{7}{|c|}{$\longrightarrow \mathrm{m}^{3} \mathrm{~m}^{-3}$} & \multicolumn{3}{|c|}{$\mathrm{kg} \mathrm{dm}^{-3}$} \\
\hline $0-10$ & \multicolumn{2}{|c|}{0,10} & \multicolumn{3}{|c|}{0,25} & \multicolumn{2}{|c|}{0,35} & \multicolumn{3}{|c|}{1,52} \\
\hline $10-20$ & \multirow{2}{*}{\multicolumn{2}{|c|}{$\begin{array}{l}0,08 \\
0,07\end{array}$}} & \multirow{2}{*}{\multicolumn{3}{|c|}{$\begin{array}{l}0,25 \\
0,26\end{array}$}} & & 0,33 & \multirow{2}{*}{\multicolumn{3}{|c|}{$\begin{array}{l}1,69 \\
1,74\end{array}$}} \\
\hline \multirow[t]{2}{*}{$20-40$} & & & & & & & 0,33 & & & \\
\hline & $\begin{array}{l}\mathbf{P}_{\text {resina }} \\
\mathrm{mg} \mathrm{dm}^{-3}\end{array}$ & $\begin{array}{l}\text { MO } \\
\mathrm{g} \mathrm{dm}^{-3}\end{array}$ & pH $\mathrm{CaCl}_{2}$ & $\mathbf{K}^{+}$ & $\mathrm{Ca}^{2+}$ & $\mathrm{Mg}^{2+}$ & $\begin{array}{l}\mathrm{H}+\mathrm{Al} \\
\mathrm{ol}_{\mathrm{c}} \mathrm{dm}^{-3}\end{array}$ & SB & CTC & $\begin{array}{l}\mathbf{V} \\
\%\end{array}$ \\
\hline $0-20$ & 1 & 7,0 & 4,0 & 0,2 & 2,0 & 1,0 & 20,0 & 3,2 & 23,2 & 14 \\
\hline $20-40$ & 0 & 4,0 & 4,2 & 0,2 & 2,0 & 1,0 & 20,0 & 3,2 & 23,2 & 14 \\
\hline
\end{tabular}


foi de 10 sementes $\mathrm{m}^{-1}$, com espaçamento de $0,50 \mathrm{~m}$ entre linhas. No início do florescimento, as plantas dos adubos verdes eram roçadas e deixadas na superfície.

Em 1996 foi realizada novamente a correção do solo, com base na análise química do solo (Quadro 2), para os tratamentos que receberam calcário e calcário mais gesso. A substituição de $\mathrm{CaO}$ do calcário pelo $\mathrm{CaO}$ do gesso seguiu o mesmo procedimento do início do trabalho, isto é, houve substituição de $25 \%$ de $\mathrm{CaO}$ do calcário pelo equivalente a $18,5 \%$ de Ca do gesso. A correção foi feita nos casos em que a saturação por bases estava inferior a $60 \%$, objetivando-se elevá-la a $70 \%$.

Em janeiro de 1997, foi semeada em toda a área experimental a cultura do milho (Zea mays L.), utilizando a semente AG 405 e $250 \mathrm{~kg}^{\mathrm{ha}}{ }^{-1}$ da fórmula 4-30-10 mais 0,3 \% de Zn, sem aplicação de calcário. Em junho de 1998, foi semeada aveia-preta (Avena strigosa Schieb.). Em fevereiro de 1999, foi semeada Brachiaria decumbens Stapf., com o intuito de proteger o solo e aumentar o seu conteúdo de matéria orgânica. Em 2006, começaram a aparecer naturalmente espécies arbóreas nativas de Cerrado.

O delineamento experimental utilizado foi inteiramente casualizado, constando de nove tratamentos (Quadro 3) e quatro repetições.

Foi avaliada a distribuição e estabilidade de agregados estáveis em água, segundo método de Angers
\& Mehuys (2000). As amostras indeformadas (torrão) foram coletadas com enxadão nas camadas de solo de 0-10, 10-20 e 20-40 cm. Os agregados foram levados para o laboratório e separados em peneiras com abertura de 6-4 mm. Para a análise, foram utilizadas as classes de agregados com tamanhos de $6-4 ; 4-2$; $2-1 ; 1-0,50 ; 0,50-0,25$; e $<0,25 \mathrm{~mm}$.

Também foi avaliado o teor de matéria orgânica do solo pelo método colorimétrico, de acordo com Raij \& Quaggio (1983), nas mesmas camadas de solo descritas anteriormente.

Com os dados, realizaram-se a análise de variância (ANOVA) e o teste de Tukey para as comparações de médias a $5 \%$. Foi usado o programa computacional SISVAR (Ferreira, 2003) para realização da análise estatística.

\section{RESULTADOS E DISCUSSÃO}

Para a distribuição e estabilidade de agregados em água e DMP, na camada de $0-10 \mathrm{~cm}$, o teste $\mathrm{F}$ foi significativo, exceto para a classe de agregados com diâmetro entre 4 e 2 mm (Quadro 4). Verificou-se que, para a percentagem de estabilidade de agregados referente ao diâmetro de 4-2 $\mathrm{mm}$, os tratamentos de recuperação do solo agiram de forma semelhante para essa propriedade física do solo. Kiehl (1979) afirma que a incorporação de resíduos orgânicos no solo provoca

Quadro 2. Valores médios dos atributos químicos do solo dos tratamentos que receberam aplicação de calcário e gesso (1996)

\begin{tabular}{|c|c|c|c|c|c|c|c|c|c|c|}
\hline Profundidade & $\mathbf{P}_{\text {resina }}$ & MO & pH CaCl${ }_{2}$ & $\mathbf{K}$ & $\mathbf{C a}$ & $\mathbf{M g}$ & $\mathbf{H}+\mathbf{A l}$ & SB & CTC & V \\
\hline $\mathrm{cm}$ & $\mathrm{mg} \mathrm{dm}^{-3}$ & $\mathrm{~g} \mathrm{dm}^{-3}$ & & & & $-\mathrm{m}$ & $\mathrm{dm}^{-3}-$ & & - & $\%$ \\
\hline $0-20$ & 1 & 1,8 & 4,7 & 0,1 & 7,7 & 3,6 & 15,0 & 11,4 & 26,4 & 43 \\
\hline $20-40$ & 1 & 1,7 & 4,4 & 0,1 & 5,0 & 2,9 & 16,0 & 8,0 & 24,0 & 33 \\
\hline
\end{tabular}

Quadro 3. Tratamentos utilizados na área experimental (1992-2008)

\begin{tabular}{|c|c|c|c|c|c|}
\hline \multirow{2}{*}{ Tratamento } & \multicolumn{2}{|c|}{ Tratamentos } & \multicolumn{3}{|c|}{ Culturas } \\
\hline & $(1992-1994)$ & (1994-1997) & 1997 & 1998 & $(1999-2008)$ \\
\hline $\mathrm{SM} / \mathrm{B}$ & $\begin{array}{l}\text { Solo mobilizado (somente operações } \\
\text { mecanizadas, sem cultura) }\end{array}$ & $\begin{array}{l}\text { Solo mobilizado (somente operações } \\
\text { mecanizadas, sem cultura) }\end{array}$ & milho & aveia-preta & braquiária \\
\hline $\mathrm{MP} / \mathrm{B}$ & Mucuna-preta & Mucuna-preta & milho & aveia-preta & braquiária \\
\hline $\mathrm{G} / \mathrm{FP} / \mathrm{B}$ & Guandu & Feijão-de-porco & milho & aveia-preta & braquiária \\
\hline $\mathrm{C}+\mathrm{MP} / \mathrm{B}$ & Calcário+Mucuna-preta & Calcário+Mucuna-preta & milho & aveia-preta & braquiária \\
\hline $\mathrm{C}+\mathrm{G} / \mathrm{FP} / \mathrm{B}$ & Calcário+Guandu & Feijão-de-porco & milho & aveia-preta & braquiária \\
\hline $\mathrm{C}+\mathrm{Ge}+\mathrm{MP} / \mathrm{B}$ & Calcário+Gesso+Mucuna-preta & Calc.+Gesso+M.preta & milho & aveia-preta & braquiária \\
\hline $\mathrm{C}+\mathrm{Ge}+\mathrm{G} / \mathrm{FP} / \mathrm{B}$ & Calcário+Gesso+Guandu & Feijão-de-porco & milho & aveia-preta & braquiária \\
\hline MA & Vegetação nativa de Cerrado. & & & & \\
\hline $\mathrm{SE}$ & Solo exposto (sem técnica de recuperação) & & & & \\
\hline
\end{tabular}

Mucuna-preta: Stizolobium aterrimum (Piper \& Tracy); guandu: Cajanus cajan (L. Millsp); feijão-de-porco: Canavalia ensiformis (L. DC); milho: Zea mays (L.); aveia-preta: Avena strigosa (Schieb.); braquiária: Brachiaria decumbens (Stapf.). 
intensa atividade de microrganismos, os quais agem como cimentantes das partículas, pelos seus micélios ou pelas substâncias viscosas produzidas - efeito este verificado neste trabalho.

As testemunhas (vegetação nativa do Cerrado e solo exposto) apresentaram maior e menor percentagem de distribuição de agregados com o diâmetro entre 6 e $4 \mathrm{~mm}$, respectivamente. Esse comportamento ocorreu devido ao fato de o solo estar sob condição natural, portanto sem intervenção pelo homem (teor médio de matéria orgânica), e, no segundo caso, pelo fato de o solo estar degradado (baixo teor de matéria orgânica, baixa estabilidade de agregados). Para as classes de diâmetro de $4-2 ; 2-1 ; 1-0,5 ; 0,5-$ 0,$25 ; \mathrm{e}<0,25 \mathrm{~mm}$, não houve diferença significativa entre os tratamentos.

Resultados de pesquisas encontrados por Campos (2006) e Bonini et al. (2007), que trabalharam em áreas degradadas semelhantes às desta pesquisa, são coincidentes em termos de comportamento com os obtidos no presente estudo. Esses pesquisadores, trabalhando com recuperação de áreas degradadas, verificaram aumento no DMP quando foram adicionados resíduos orgânicos. Eles destacam ainda que o papel da matéria orgânica na agregação do solo é um processo dinâmico, sendo necessário o acréscimo contínuo de material orgânico para manter a estrutura adequada ao desenvolvimento das plantas (Campos et al., 1995).

Analisando a classe de agregados de 6-4 mm, os tratamentos com guandu e as combinações com calcário e gesso, e o tratamento com mucuna com calcário e gesso, bem como a mucuna-preta isolada (sem calcário e gesso), foram semelhantes na estabilidade de agregados para a condição de solo sob Cerrado (Quadro 4), com maior percentagem de macroagregados estáveis.

Em relação ao DMP (diâmetro médio ponderado), a vegetação nativa do Cerrado apresentou maior valor, e os tratamentos que foram semelhantes estatisticamente nas condições do solo sob vegetação do Cerrado foram: MP/B, C+G/FP/B e C+Ge+MP/B (Quadro 4). Esses dados, quando comparados com os de Andrade Júnior (2004), que trabalhou na mesma área experimental em 2004, mostram que houve incremento de até $2,00 \mathrm{~mm}$ nos tratamentos de recuperação e que os adubos verdes agiram ao longo do tempo. Esses efeitos também foram verificados por Taboada-Castro (2009), Kitamura (2007), Campos (2006) e Colodro (2005), que estudaram a recuperação de áreas degradadas e utilizaram a estabilidade de agregados em água como indicador de qualidade do solo.

O experimento encontra-se desde 1999 com $B$. decumbens em todas as parcelas; essa cobertura protege o solo contra a desagregação pelo impacto da chuva e variações bruscas de umidade. Ao mesmo tempo, o contínuo fornecimento de material orgânico, quer por secreções radiculares, quer por renovação do sistema radicular e da parte aérea ou dos resíduos de colheita, serve de fonte de energia para a atividade microbiana, cujos subprodutos, constituídos de moléculas orgânicas em diversas fases de decomposição, atuam como agentes de formação e estabilização dos agregados. Ao lado desses benefícios, manifesta-se também o efeito mecânico e físico das raízes sobre a formação dos agregados (Alves, 2001).

Na camada de $10-20 \mathrm{~cm}$, o teste $\mathrm{F}$ foi significativo para as classes de agregados com diâmetros de 6-4; $4-2 ; 1-0,5 ; 0,5-0,25 ;<0,25$; e DMP (Quadro 4). Somente para a classe de tamanho de $2-1 \mathrm{~mm}$ não houve diferença significativa. Nesta camada, os valores encontrados para a percentagem de distribuição de agregados estáveis referente à classe com diâmetro de 6-4 $\mathrm{mm}$ foram semelhantes entre a testemunha (vegetação nativa do Cerrado) e o tratamento $\mathrm{C}+\mathrm{Ge}+\mathrm{G} / \mathrm{FP} / \mathrm{B}$. Para as demais classes de diâmetro de agregados, a distribuição deles foi semelhante entre os tratamentos usados para a recuperação do solo, diferindo do solo sob vegetação natural. Esses resultados concordam com os de Andrade Júnior (2004), que, trabalhando nessa mesma área experimental, não verificou efeito nos tratamentos usados para recuperação do solo. Os valores indicam que o solo não está recuperando suas propriedades nesta camada, pelo baixo valor de macroagregados $(6-4 \mathrm{~mm})$ e pela maior quantidade de microagregados.

Kitamura (2007) lembra que um solo com boa estrutura apresenta boa estabilidade de agregados, porém nem todo solo com boa estabilidade de agregados possui boa estrutura, sendo necessário conhecer o histórico da área para que não ocorram equívocos de interpretação. Carpenedo \& Mielniczuk (1990) mencionam que esse comportamento pode estar relacionado com a maior coesão dos macroagregados proporcionada pela aproximação das partículas em operações de preparo, considerados agregados de menor qualidade, ou pelo efeito negativo de ciclos intensos de secagem e umedecimento, enfraquecendo as ligações entre partículas na camada superficial.

Na camada de 20-40 cm (Quadro 4) não houve significância para o teste $\mathrm{F}$ somente para a classe de agregados com diâmetro de $2-1 \mathrm{~mm}$ - comportamento semelhante ao da camada de 10-20 cm. Comparando os tratamentos usados para recuperação do solo e a testemunha (solo sob Cerrado) pelo teste de Tukey a $5 \%$, somente a vegetação nativa do Cerrado foi diferente dos demais tratamentos. Nesta camada de solo, os tratamentos de recuperação influenciaram a estabilidade de agregados de forma semelhante. $\mathrm{O}$ valor obtido para o diâmetro de $6-4 \mathrm{~mm}$ na vegetação nativa do Cerrado mostra a superioridade em relação aos tratamentos usados para recuperação do solo. Indica ainda que não houve incremento no teor de matéria orgânica nesta camada (Quadro 5), promovendo efeitos sobre a agregação, pois Alves (2001) menciona que a influência da matéria orgânica na 
agregação do solo é um processo dinâmico: à medida que se adiciona material orgânico ao solo, a atividade é intensificada, resultando em produtos que desempenham função na formação e estabilização (agentes cimentantes) dos agregados.
Os tratamentos usados para recuperação agiram na camada superficial do solo; contudo, comparando o DMP com o do experimento realizado nessa mesma área experimental por Andrade Júnior (2004), podese verificar aumento no DMP em todos os tratamentos

Quadro 4. Teste F, diferença mínima significativa (DMS) a $5 \%$, coeficiente de variação (CV) e valores médios para a \% de distribuição de agregados para as classes de tamanho de agregados e DMP (mm) nas camadas de 0-10, 10-20 e 20-40 cm (2008)

\begin{tabular}{|c|c|c|c|c|c|c|c|}
\hline \multirow{2}{*}{ Tratamento } & \multicolumn{7}{|c|}{ Classe de tamanho de agregados (mm) } \\
\hline & $6-4$ & $4-2$ & $2-1$ & $1-0,5$ & $0,5-0,25$ & $<0,25$ & DMP \\
\hline & & & & $-0-10 \mathrm{~cm}$ & & & \\
\hline $\mathrm{F}$ & $6,88^{*}$ & $0,82^{\text {ns }}$ & $8,98^{*}$ & $9,03^{*}$ & $10,53^{*}$ & $12,45^{*}$ & $8,83^{*}$ \\
\hline DMS (5 \%) & 2,423 & 1,139 & 0,906 & 1,251 & 1,034 & 1,502 & 0,345 \\
\hline CV (\%) & 23,62 & 17,92 & 21,42 & 26,23 & 24,64 & 24,04 & 11,84 \\
\hline $\mathrm{SM} / \mathrm{B}$ & $29,4 \mathrm{AB}$ & $16,6 \mathrm{~A}$ & $9,3 \mathrm{~B}$ & $12,1 \mathrm{BC}$ & $8,9 \mathrm{BC}$ & $23,6 \mathrm{BC}$ & $2,3 \mathrm{AB}$ \\
\hline $\mathrm{MP} / \mathrm{B}$ & $53,4 \mathrm{BC}$ & $13,6 \mathrm{~A}$ & $6,8 \mathrm{~B}$ & $8,3 \mathrm{BC}$ & $5,7 \mathrm{~B}$ & $12,2 \mathrm{~B}$ & $3,3 \mathrm{BC}$ \\
\hline $\mathrm{G} / \mathrm{FP} / \mathrm{B}$ & $28,0 \mathrm{AB}$ & $17,2 \mathrm{~A}$ & $10,7 \mathrm{~B}$ & $14,4 \mathrm{BC}$ & $9,7 \mathrm{BC}$ & $20,0 \mathrm{BC}$ & $2,2 \mathrm{AB}$ \\
\hline $\mathrm{C}+\mathrm{MP} / \mathrm{B}$ & $38,8 \mathrm{AB}$ & $13,6 \mathrm{~A}$ & $8,3 \mathrm{~B}$ & $11,8 \mathrm{BC}$ & $8,1 \mathrm{BC}$ & $19,4 \mathrm{BC}$ & $2,6 \mathrm{AB}$ \\
\hline $\mathrm{C}+\mathrm{G} / \mathrm{FP} / \mathrm{B}$ & $50,4 \mathrm{BC}$ & $17,9 \mathrm{~A}$ & $5,6 \mathrm{~B}$ & $6,7 \mathrm{~B}$ & $5,7 \mathrm{~B}$ & $13,6 \mathrm{~B}$ & $3,2 \mathrm{BC}$ \\
\hline $\mathrm{C}+\mathrm{Ge}+\mathrm{MP} / \mathrm{B}$ & $50,1 \mathrm{BC}$ & $18,0 \mathrm{~A}$ & $6,5 \mathrm{~B}$ & $6,8 \mathrm{~B}$ & $5,7 \mathrm{~B}$ & $12,9 \mathrm{AB}$ & $3,2 \mathrm{BC}$ \\
\hline $\mathrm{C}+\mathrm{Ge}+\mathrm{G} / \mathrm{FP} / \mathrm{B}$ & $49,0 \mathrm{BC}$ & $14,5 \mathrm{~A}$ & $6,3 \mathrm{~B}$ & $8,6 \mathrm{BC}$ & $6,8 \mathrm{~B}$ & $14,8 \mathrm{~B}$ & $3,1 \mathrm{~B}$ \\
\hline MA & $80,0 \mathrm{C}$ & $15,8 \mathrm{~A}$ & $1,1 \mathrm{~A}$ & $0,9 \mathrm{~A}$ & $0,5 \mathrm{~A}$ & $1,6 \mathrm{~A}$ & $4,5 \mathrm{C}$ \\
\hline $\mathrm{SE}$ & $17,4 \mathrm{~A}$ & $13,5 \mathrm{~A}$ & $8,7 \mathrm{~B}$ & $15,6 \mathrm{C}$ & $13,4 \mathrm{C}$ & $31,4 \mathrm{C}$ & $1,6 \mathrm{~A}$ \\
\hline & & & & $-10-20 \mathrm{~cm}$ & & & \\
\hline $\mathrm{F}$ & $5,562^{*}$ & $4,984^{*}$ & $1,753^{\mathrm{ns}}$ & $7,711^{*}$ & $11,742^{*}$ & $10,181^{*}$ & $7,280^{*}$ \\
\hline DMS (5\%) & 2,995 & 1,033 & 7,537 & 1,452 & 1,155 & 1,461 & 0,409 \\
\hline CV (\%) & 43,35 & 18,15 & 46,21 & 21,86 & 20,33 & 19,57 & 16,82 \\
\hline $\mathrm{SM} / \mathrm{B}$ & $9,6 \mathrm{~A}$ & $12,2 \mathrm{~A}$ & $12,1 \mathrm{~A}$ & $21,7 \mathrm{BC}$ & $15,8 \mathrm{BC}$ & $28,5 \mathrm{~B}$ & $1,3 \mathrm{~A}$ \\
\hline $\mathrm{MP} / \mathrm{B}$ & $29,4 \mathrm{~A}$ & $8,6 \mathrm{~A}$ & $8,5 \mathrm{~A}$ & $15,8 \mathrm{~B}$ & $13,4 \mathrm{BC}$ & $24,3 \mathrm{~B}$ & $2,0 \mathrm{~A}$ \\
\hline $\mathrm{G} / \mathrm{FP} / \mathrm{B}$ & $12,0 \mathrm{~A}$ & $9,9 \mathrm{~A}$ & $12,7 \mathrm{~A}$ & $19,5 \mathrm{BC}$ & $16,2 \mathrm{BC}$ & $29,6 \mathrm{~B}$ & $1,3 \mathrm{~A}$ \\
\hline $\mathrm{C}+\mathrm{MP} / \mathrm{B}$ & $19,8 \mathrm{~A}$ & $11,9 \mathrm{~A}$ & $11,1 \mathrm{~A}$ & $18,2 \mathrm{BC}$ & $13,8 \mathrm{BC}$ & $25,1 \mathrm{~B}$ & $1,7 \mathrm{~A}$ \\
\hline $\mathrm{C}+\mathrm{G} / \mathrm{FP} / \mathrm{B}$ & $16,6 \mathrm{~A}$ & $13,0 \mathrm{~A}$ & $10,8 \mathrm{~A}$ & $21,3 \mathrm{BC}$ & $13,4 \mathrm{BC}$ & $24,8 \mathrm{~B}$ & $1,6 \mathrm{~A}$ \\
\hline $\mathrm{C}+\mathrm{Ge}+\mathrm{MP} / \mathrm{B}$ & $17,8 \mathrm{~A}$ & $13,5 \mathrm{AB}$ & $11,4 \mathrm{~A}$ & $17,2 \mathrm{BC}$ & $13,3 \mathrm{BC}$ & $26,8 \mathrm{~B}$ & $1,7 \mathrm{~A}$ \\
\hline $\mathrm{C}+\mathrm{Ge}+\mathrm{G} / \mathrm{FP} / \mathrm{B}$ & $31,6 \mathrm{AB}$ & $12,4 \mathrm{~A}$ & $10,2 \mathrm{~A}$ & $16,1 \mathrm{BC}$ & $9,7 \mathrm{~B}$ & $19,9 \mathrm{~A}$ & $2,3 \mathrm{~A}$ \\
\hline MA & $62,1 \mathrm{~B}$ & $22,4 \mathrm{~B}$ & $5,7 \mathrm{~A}$ & $4,0 \mathrm{~A}$ & $1,8 \mathrm{~A}$ & $4,0 \mathrm{~B}$ & $3,9 \mathrm{~B}$ \\
\hline $\mathrm{SE}$ & $9,9 \mathrm{~A}$ & $10,0 \mathrm{~A}$ & $8,7 \mathrm{~A}$ & $28,3 \mathrm{C}$ & $21,0 \mathrm{C}$ & $22,0 \mathrm{~B}$ & $1,3 \mathrm{~A}$ \\
\hline & & & $145 \mathrm{~ns}$ & Cm & & & \\
\hline $\mathrm{F}$ & $12,83^{*}$ & $7,37^{*}$ & $1,553^{\mathrm{ns}}$ & $4,882^{*}$ & $10,630^{*}$ & $10,335^{*}$ & $16,695^{*}$ \\
\hline DMS (5 \%) & 1,859 & 0,953 & 0,917 & 1,123 & 0,803 & 1,117 & 0,242 \\
\hline $\mathrm{CV}(\%)$ & 44,53 & 21,19 & 16,72 & 14,13 & 11,90 & 13,07 & 11,90 \\
\hline $\mathrm{SM} / \mathrm{B}$ & $2,1 \mathrm{~A}$ & $5,1 \mathrm{~A}$ & $9,7 \mathrm{~A}$ & $27,9 \mathrm{~B}$ & $21,2 \mathrm{~B}$ & $34,0 \mathrm{~B}$ & $0,7 \mathrm{~A}$ \\
\hline $\mathrm{MP} / \mathrm{B}$ & $13,2 \mathrm{~A}$ & $7,2 \mathrm{~A}$ & $9,4 \mathrm{~A}$ & $24,0 \mathrm{~B}$ & $18,4 \mathrm{~B}$ & $27,8 \mathrm{~B}$ & $1,3 \mathrm{~A}$ \\
\hline $\mathrm{G} / \mathrm{FP} / \mathrm{B}$ & $2,7 \mathrm{~A}$ & $5,7 \mathrm{~A}$ & $9,7 \mathrm{~A}$ & $23,9 \mathrm{~B}$ & $19,7 \mathrm{~B}$ & $38,3 \mathrm{~B}$ & $0,7 \mathrm{~A}$ \\
\hline $\mathrm{C}+\mathrm{MP} / \mathrm{B}$ & $11,7 \mathrm{~A}$ & $9,7 \mathrm{~A}$ & $13,7 \mathrm{~A}$ & $23,1 \mathrm{~B}$ & $15,1 \mathrm{~B}$ & $26,8 \mathrm{~B}$ & $1,3 \mathrm{~A}$ \\
\hline $\mathrm{C}+\mathrm{G} / \mathrm{FP} / \mathrm{B}$ & $5,9 \mathrm{~A}$ & $6,5 \mathrm{~A}$ & $11,1 \mathrm{~A}$ & $25,3 \mathrm{~B}$ & $18,7 \mathrm{~B}$ & $32,507 \mathrm{~B}$ & $0,9 \mathrm{~A}$ \\
\hline $\mathrm{C}+\mathrm{Ge}+\mathrm{MP} / \mathrm{B}$ & $2,0 \mathrm{~A}$ & $5,9 \mathrm{~A}$ & $11,1 \mathrm{~A}$ & $29,9 \mathrm{~B}$ & $20,6 \mathrm{~B}$ & $30,5 \mathrm{~B}$ & $0,8 \mathrm{~A}$ \\
\hline $\mathrm{C}+\mathrm{Ge}+\mathrm{G} / \mathrm{FP} / \mathrm{B}$ & $4,8 \mathrm{~A}$ & $7,0 \mathrm{~A}$ & $12,6 \mathrm{~A}$ & $26,4 \mathrm{~B}$ & $19,0 \mathrm{~B}$ & $30,2 \mathrm{~B}$ & $0,9 \mathrm{~A}$ \\
\hline MA & $37,9 \mathrm{~B}$ & $17,4 \mathrm{~B}$ & $13,7 \mathrm{~A}$ & $12,9 \mathrm{~A}$ & $7,2 \mathrm{~A}$ & $10,9 \mathrm{~A}$ & $2,8 \mathrm{~B}$ \\
\hline $\mathrm{SE}$ & $3,8 \mathrm{~A}$ & $6,9 \mathrm{~A}$ & $12,7 \mathrm{~A}$ & $28,9 \mathrm{~B}$ & $19,6 \mathrm{~B}$ & $28,0 \mathrm{~B}$ & $0,9 \mathrm{~A}$ \\
\hline
\end{tabular}

SM/B: Solo mobilizado até 1999, após implantado Braquiária; MP/B: Mucuna-preta até 1999 após substituído por Braquiária; G/ FP/B: Guandu até 1994, após substituído por Feijão-de-porco e partir de 1999 substituido por Braquiária; C+MP/B: Calcário+Mucuna-preta até 1999, após substituído por Braquiária; C+G/FP/B: Calcário+Guandu até 1994, após substituído por Feijão-de-porco e partir de 1999 substituído por Braquiária; C+Ge+MP/B: Calcário+Gesso+Mucuna-preta até 1999 após substituído por Braquiária; C+Ge+G/FP/B: Calcário+Gesso+Guandu, até 1994, após substituído por Feijão-de-porco e partir de 1999 substituído por Braquiária; MA: Vegetação nativa do Cerrado; SE: Solo Exposto. Médias seguidas de letras iguais na coluna não diferem entre si pelo teste de Tukey a $5 \%$. Dados transformados $(\sqrt{\mathrm{x}+0,5})$. 
Quadro 5. Teste F, coeficiente de variação (CV) e valores médios para o teor de matéria orgânica nas camadas de 0-10, 10-20 e 20-40 cm (2008)

\begin{tabular}{lccc}
\hline \multirow{2}{*}{ Tratamento } & \multicolumn{3}{c}{ Camadas de solo (cm) } \\
\cline { 2 - 4 } & $\mathbf{0 - 1 0}$ & $\mathbf{1 0 - 2 0}$ & $\mathbf{0 - 4 0}$ \\
\cline { 2 - 4 } & \multicolumn{4}{c}{$\mathrm{g} \mathrm{dm}^{-3}$} \\
$\mathrm{SM} / \mathrm{B}$ & $7 \mathrm{~A}$ & 5 & 2 \\
$\mathrm{MP} / \mathrm{B}$ & $\mathrm{AB}$ & 8 & 2 \\
$\mathrm{G} / \mathrm{FP} / \mathrm{B}$ & $8 \mathrm{~A}$ & 5 & 2 \\
$\mathrm{C}+\mathrm{MP} / \mathrm{B}$ & $9 \mathrm{~A}$ & 7 & 2 \\
$\mathrm{C}+\mathrm{G} / \mathrm{FP} / \mathrm{B}$ & $6 \mathrm{~A}$ & 5 & 3 \\
$\mathrm{C}+\mathrm{Ge}+\mathrm{MP} / \mathrm{B}$ & $7 \mathrm{~A}$ & 5 & 2 \\
$\mathrm{C}+\mathrm{Ge}+\mathrm{G} / \mathrm{FP} / \mathrm{B}$ & $15 \mathrm{~B}$ & 10 & 2 \\
$\mathrm{MA}$ & $6 \mathrm{~A}$ & 5 & 3 \\
$\mathrm{SE}$ & $3,055^{*}$ & $1,496^{\mathrm{ns}}$ & 2 \\
$\mathrm{~F}$ & 15,96 & 20,24 & $1,671^{\text {ns }}$ \\
$\mathrm{CV}(\%)$ &
\end{tabular}

Dados transformados $(\sqrt{\mathrm{x}+0,5})$. SM/B: Solo mobilizado até 1999, após implantado Braquiária; MP/B: Mucuna-preta até 1999 após substituído por Braquiária; G/FP/B: Guandu até 1994, após substituído por Feijão-de-porco e partir de 1999 substituído por Braquiária; C+MP/B: Calcário+Mucuna-preta até 1999, após substituído por Braquiária; C+G/FP/B: Calcário+Guandu até 1994, após substituído por Feijão-de-porco e partir de 1999 substituído por Braquiária; $\mathrm{C}+\mathrm{Ge}+\mathrm{MP} / \mathrm{B}$ : Calcário+Gesso+ Mucuna-preta até 1999 após substituído por Braquiária; C+Ge+G/FP/B: Calcário+Gesso+Guandu, até 1994, após substituído por Feijão-de-porco e partir de 1999 substituído por Braquiária; MA: Vegetação nativa do Cerrado; SE: Solo Exposto. Médias seguidas de letras iguais na coluna não diferem entre si pelo teste de Tukey a $5 \%$.

testados, em todas as camadas de solo. O efeito positivo da adição de resíduos orgânicos foi verificado neste experimento; na camada superficial, onde o teor de matéria orgânica foi maior, a percentagem de macroagregados também foi maior (Quadro 5).

Quanto aos tratamentos de recuperação, não houve diferença entre eles; esse comportamento mostra que a adição de adubo verde e as combinações calcário ou calcário+gesso estão influenciando de forma semelhante o teor de matéria orgânica do solo. Outro fator a considerar é a cultura da braquiária, que foi instalada na área em 1999 e pode estar influenciando de forma homogênea. As gramíneas são importantes na adição de matéria orgânica e na reestruturação do solo (Assad, 1997), o que confirma dados encontrados para a estabilidade de agregados na camada superficial (Quadros 4 a 6). Colodro et al. (2007) relatam a influência do teor de matéria orgânica no solo e na atividade microbiana.

Alves et al. (2007) mostram a correlação da matéria orgânica com algumas propriedades físicas do solo. A matéria orgânica tem forte relação com a estruturação do solo, pois ela é fonte de energia para a atividade biológica do solo; em razão do fornecimento de matéria orgânica para o solo, haverá maior atividade biológica e, consequentemente, maior reestruturação.

\section{CONCLUSÕES}

1. A estrutura do solo vem sendo recuperada, e os tratamentos com uso de guandu/feijão-de-porco/ braquiária com calcário ou calcário+gesso, bem como o tratamento somente com mucuna-preta, foram os mais eficazes.

2. As modificações na melhoria da qualidade da estrutura estão ocorrendo na camada de 0-20 cm.

3. Os tratamentos agiram de forma semelhante na melhoria da qualidade da estrutura nas camadas de $10-20$ e $20-40 \mathrm{~cm}$, porém na de $10-20 \mathrm{~cm}$ o tratamento com guandu/feijão-de-porco/braquiária e calcário+gesso foi o mais promissor, assemelhando-se ao solo sob condição natural de Cerrado.

4. Em todas as camadas de solo estudadas, o solo exposto apresentou a pior condição de estrutura, comparada à do solo sob condição natural de Cerrado.

5. Quanto à camada de $20-40 \mathrm{~cm}$, conclui-se que o solo ainda se encontra com a estrutura degradada.

\section{AGRADECIMENTO}

À FAPESP, pelo apoio financeiro.

\section{LITERATURA CITADA}

ALCÂNTARA, F.A.; FURTINI NETO, A.E.; PAULA, M.B.; MESQUITA, H.A. \& MUNIZ, J.A. Adubação verde na recuperação da fertilidade. Pesq. Agropec. Bras., 35:277$88,2000$.

ALVES, M.C.; SUZUKI, L.G.A.S. \& SUZUKI, L.E.A.S. Densidade do solo e infiltração de água como indicadores da qualidade física de um Latossolo Vermelho distrófico em recuperação. R. Bras. Ci. Solo, 31:617-625, 2007.

ALVES, M.C. Recuperação do subsolo de um Latossolo Vermelho usado para terrapleno e fundação da usina hidrelétrica de Ilha Solteira - SP. Ilha Solteira, Universidade Estadual Paulista 2001. 83p. (Tese de Livre Docência)

ANDRADE JÚNIOR, R.T. Propriedades Físico-químicas de um solo em recuperação e adaptação da Brachiaria decumbens. Ilha Solteira, Universidade Estadual Paulista, 2004. 49p. (Trabalho de Graduação)

ANGERS, D.A. \& MEHUYS, G.R. Aggregate stability to water. In: CARTER, M.R. Soil sampling and methods of analysis. Boca Raton, Canadian Society of Soil Science/Lewis Publishers, 2000.

ASSAD, M.L.L. Fauna do solo. In: VARGAS, M.A.T. \& HUNGRIA, M. Biologia dos solos dos Cerrados. Planaltina, Embrapa, 1997. 524p. 
AVARETTO, N.; MORAES, A.; MOTTA, A.C.V. \& PREVEDELLO, B.M.S. Efeito da revegetação e da adubação de área degradada na fertilidade do solo e nas características da palhada. Pesq. Agropec. Bras., 35:289$297,2000$.

BITAR, O.Y. Avaliação da recuperação de áreas degradadas por mineração na região Metropolitana de São Paulo. São Paulo, 1997. 185p.

BONINI, C.S.B.; OLIVEIRA, B.A. \& ALVES, M.C. Estabilidade de agregados de um Latossolo Vermelho tratado com lodo de esgoto e adubação mineral In: SIMPÓSIO INTERNACIONAL DE INICIAÇÃO CIENTIFICA DA USP, 15., Pirassununga, 2007. Anais... Pirassununga, ESALQ- USP, 2007. p.1-1.

CAMPOS, B.C.; REINERT, D.J.; NICOLODI, R.; RUEDELL, J. \& PETRERE, C. Estabilidade estrutural de um Latossolo Vermelho-Escuro distrófico após sete anos de rotação de culturas e sistemas de manejo de solo. R. Bras. Ci. Solo, 19:121-126, 1995.

CAMPOS, F.S. \& ALVES, M.C. Uso de lodo de esgoto na reestruturação de solo degradado. R. Bras. Ci. Solo, 32:1389-1397, 2008.

CAMPOS, F.S. Uso de lodo de esgoto na reestruturação de Latossolo Vermelho degradado. Ilha Solteira, Universidade Estadual Paulista, 2006. 106p. (Tese de Mestrado)

COLODRO, G.; ESPINDOLA, C. R.; CASSIOLATO, A.M.R. \& ALVES, M.C. Atividade microbiana em um Latossolo degradado tratado com lodo de esgoto. R. Bras. Eng. Agríc. Amb., 11:195-198, 2007.

COLODRO, G. Recuperação de solo de área de empréstimo com lodo de esgoto. Campinas, Universidade Estadual de Campinas, 2005. 82p. (Tese de Doutorado)

CARPENEDO, V. \& MIELNICZUK, J. Estado de agregação e qualidade de agregados de Latossolos Roxos, submetidos a diferentes sistemas de manejo. R. Bras. Ci. Solo, 14:99$105,1990$.

DEMATTÊ, J.L.I. Levantamento detalhado dos solos do Campus Experimental de Ilha Solteira (SP). Piracicaba, 1980. 131p. (Mimeografado)

EMPRESA BRASILEIRA DE PESQUISA AGROPECUÁRIA . EMBRAPA. Sistema brasileiro de classificação de solos. 2.ed. Rio de Janeiro, Embrapa, 2006. 306p.
FAO. World reference base for soil resources. Rome, FAO, 1998. (World Soil Resources Report, 60)

FERREIRA, D.F. Sisvar versão 4.2. Lavras, DEX/UFLA, 2003.

JAENICKE, E.C. From the ground up: Exploring soil quality's contribution to environmental health. In: LOPES, E.B.M. Diversidade metabólica em solo tratado com biossólidos. Piracicaba, Escola Superior de Agricultura Luiz de Queiroz, 2001. 65p. (Tese de Mestrado)

JASTROW, J.D.; MILLER, R.M. \& LUSSENHOP, J. Contributions of interacting biological mechanisms to soil aggregate stabilization in restored prairie. Soil Biol. Bioci., 30:905-916, 1998.

KIEHL, E.J. Manual de edafologia: Relação solo-planta. São Paulo, Agronômica Ceres, 1979. 264p.

KITAMURA, A.E. Recuperação de um subsolo de Cerrado com cultivo de plantas nativa e introduzidas. Ilha Solteira, Universidade Estadual Paulista, 2007. 113p. (Tese de Doutorado)

KITAMURA, A.E.; ALVES, M.C.; SUZUKI, L.G.A.S. \& GONZALEZ, A.P. Recuperação de um solo degradado com a aplicação de adubos verdes e lodo de esgoto. R. Bras. Ci. Solo, 32:405-416, 2008.

RAIJ, B.van \& QUAGGIO, J.A. Métodos de análises de solo para fins de fertilidade. Campinas, Instituto Agronômico de Campinas, 1983. (Boletim Técnico, 31)

ROTH, C.H.; CASTRO FILHO, C. \& MEDEIROS, G.B. Análise de fatores físicos e químicos relacionados com a agregação de um Latossolo Roxo distrófico. R. Bras. Ci. Solo, 15:241248,1991

SOIL SURVEY STAFF. Soil taxonomy: A basic system of soil classification for making and interpreting soil surveys. 2.ed. Washington, Government Printing Office, 1999. (USDA, Handbook, 436)

TABOADA-CASTRO, M.M.; ALVES, M.C.; NASCIMENTO, V. \& TABOADA-CASTRO, T. Revegetation on a removed topsoil: Effect on aggregate stability. Comm. Soil Sci. Plant Anal., 40:771-786, 2009. 